\title{
Eplerenone Versus Observation in the Treatment of Acute Central Serous Chorioretinopathy: A Retrospective Controlled Study
}

\author{
Ilaria Zucchiatti - Riccardo Sacconi - Maria Cristina Parravano - Eliana Costanzo • \\ Lea Querques · Daniela Montorio · Francesco Bandello · Giuseppe Querques
}

Received: January 3, 2018 / Published online: February 13, 2018

(C) The Author(s) 2018. This article is an open access publication

\begin{abstract}
Introduction: To investigate the effects of eplerenone compared to observation in the treatment of acute central serous chorioretinopathy (CSC).

Methods: Charts of consecutive patients with a diagnosis of acute CSC (visual symptoms $<12$ weeks) were reviewed. Included patients were divided into a treatment group (treated with eplerenone) and a control group (observation). Main outcome measures included changes in best-corrected visual acuity (BCVA), central macular thickness (CMT), height of
\end{abstract}

Enhanced content To view enhanced content for this article go to https://doi.org/10.6084/m9.figshare. 5844888 .

I. Zucchiatti · R. Sacconi - L. Querques ·

D. Montorio · F. Bandello · G. Querques ( $₫)$

Department of Ophthalmology, University Vita-

Salute, IRCCS Ospedale San Raffaele, Milan, Italy

e-mail: giuseppe.querques@hotmail.it

R. Sacconi

Department of Neurological, Biomedical and

Movement Sciences, Eye Clinic, University of

Verona, Verona, Italy

M. C. Parravano - E. Costanzo - G. Querques Fondazione G. B. Bietti-IRCCS, Rome, Italy

D. Montorio

Department of Neurosciences, Reproductive

Sciences and Dentistry, Eye Clinic, University of

Naples Federico II, Naples, Italy subretinal fluid (SRF) and subfoveal choroidal thickness (CT) at 1 and 3 months in the two groups.

Results: Fifteen eyes of 15 patients ( 2 female, 13 male) and 12 eyes of 12 patients ( 1 female, 11 male $[p=1.000])$ were included in the treatment and control groups, respectively. The mean age was $44 \pm 9(30-65)$ and $47 \pm 11$ years (28-66 years, $p=0.493$ ), respectively. In the treatment group, BCVA improved significantly at 1 month $(p=0.018)$ and 3 months of followup $(p=0.011)$, while a non-significant improvement was seen in the control group. At 3 months, 12 of 15 eyes (80\%) in the treatment group demonstrated complete SRF resolution, versus 3 of 12 eyes (25\%) in the control group $(p=0.007)$. In the treatment group, SRF and CMT were significantly reduced at the 1 -month follow-up $(p=0.014, p=0.028$, respectively) and the 3-month follow-up $(p<0.001$ for both analyses), while in the control group the changes were not statistically significant. Eplerenone was well tolerated in all patients.

Conclusion: Patients affected by acute CSC treated with eplerenone achieved greater and faster resolution of the disease compared to the observation group. Eplerenone may represent an attractive new first-line treatment option for acute CSC.

Keywords: Central serous chorioretinopathy; Eplerenone; Optical coherence tomography; OCT angiography 


\section{INTRODUCTION}

Central serous chorioretinopathy (CSC) is a common disorder that typically affects young adult men, with an incidence of approximately 5.8 per 100,000 people [1]. Acute disease is characterized by the presence of serous retinal detachment and/or retinal pigment epithelial (RPE) detachment located in the macular region, leading to central vision loss, dyschromatopsia, central or paracentral scotoma, metamorphopsia and/or micropsia. Acute CSC is typically a benign disorder that resolves spontaneously in 2-3 months [2]. Nevertheless, approximately $30-50 \%$ of patients experience a recurrence of the disease over the long term $[3,4]$. In addition, $5-10 \%$ of cases of unresolved disease may progress to chronic CSC (also known as diffuse retinal epitheliopathy), which includes persistent serous retinal detachment, associated with diffuse RPE alterations and, in some cases, cystoid retinal degeneration or pigment epithelium detachment [3]. In chronic CSC, the persistent fluid accumulation leads to progressive and irreversible damage to the photoreceptors that may have a decisive effect on visual prognosis $[3,5]$.

The pathogenesis of CSC is extremely complex and still poor understood. Risk factors for acute CSC include a stress-prone personality (type A behavior), an increased sympathetic activity, the use of systemic corticosteroid and hypertension [6-8].

Recent investigations strongly support the hypothesis that CSC is primarily characterized by vascular deregulation in the choroid. In fact, choroidal hyperpermeability changes have been clearly shown using fluorescein angiography (FA) and indocyanine green angiography (ICGA) $[9,10]$, and increased choroidal thickness has been demonstrated in both the affected and contralateral eyes when measured with optical coherence tomography (OCT) [11-13]. New insights into the pathogenesis of this multifactorial disease have revealed that glucocorticoid and mineralocorticoid dysfunction is also present [14-16]. In animal models, the activation of choroidal mineralocorticoid receptors was shown to cause choroidal vasodilation and leakage; this phenomenon was inhibited by mineralocorticoid receptor (MR) antagonism [17]. Based on these findings, researchers investigated the role of mineralocorticoid antagonists in the treatment of CSC, reporting positive preliminary results [17-28]. Spironolactone and eplerenone are both oralspecific MR antagonists approved for several systemic disorders, and are commonly used for treating hypertension and congestive heart failure. However, spironolactone, although effective, is associated with various unwanted progestational and antiandrogenic side effects, such as gynecomastia, impotence and abnormal menstrual cycles, which can limit its use. Eplerenone has a lower affinity for steroid receptors (e.g. progesterone and androgen), leading to higher tolerability and specificity.

Recent reports have highlighted the potential benefits of eplerenone in patients with CSC, showing promising results in terms of gains in best-corrected visual acuity (BCVA) and reductions in central macular thickness (CMT) on OCT. However, to the best of our knowledge, there are no data available concerning the treatment of acute CSC with eplerenone compared to observation. Therefore, the aim of this study was to investigate the effects of eplerenone in the treatment of acute CSC compared to observation.

\section{MATERIALS AND METHODS}

We reviewed the charts of consecutive patients with a diagnosis of acute CSC referred to the Medical Retina and Imaging Division of San Raffaele Hospital (Milan, Italy) and to the Fondazione G. B. Bietti-IRCCS (Rome, Italy) between January 2016 and July 2017, treated with eplerenone or with observation. All procedures performed in studies involving human participants were in accordance with the ethical standards of the institutional research committee and with the 1964 Helsinki declaration and its later amendments or comparable ethical standards. Informed consent was obtained from all individual participants included in the study.

Patients were eligible for inclusion if they were at least 18 years of age, with a diagnosis of 
acute CSC, defined by the presence of subretinal fluid and visual symptoms for less than 12 weeks, and were experiencing an episode of CSC for the first time. Exclusion criteria were the presence of chronic CSC (duration of visual symptoms more than 12 weeks) or recurrent CSC (patients with a history of one or more previous CSC attacks), presence of choroidal neovascularization (CNV) identified by FA, ICGA and/or OCT angiography (OCT-A), any treatment for retinal disease (including intravitreal injections, photodynamic therapy, laser photocoagulation, vitrectomy), history of other retinal disorders (including age-related macular degeneration, choroidal neovascularization, diabetic retinopathy, uveitis or pathologic myopia) and the presence of any other systemic condition for which eplerenone was contraindicated (such as severe renal, cardiac or hepatic failure, pregnancy, baseline serum potassium greater than $5.0 \mathrm{mEq} / \mathrm{L}$, concomitant administration of potassium-sparing diuretics, potassium supplements, strong CYP3A4 inhibitors, angiotensin-converting enzyme inhibitors or angiotensin receptor blockers).

All patients who met the inclusion criteria were assigned to either the treatment group (treated with eplerenone) or the observation group. All patients treated with eplerenone were consecutively included at San Raffaele Hospital, while all observed subjects were consecutively included at the Fondazione G. B. Bietti-IRCCS. The follow-up period was 3 months.

The patients in the treatment group were treated with eplerenone $25 \mathrm{mg} /$ day for 1 week and then $50 \mathrm{mg} /$ day for 11 weeks. Serum potassium and creatinine assessment were performed at baseline before starting treatment and monthly during treatment. Eplerenone was discontinued in the case of increased serum potassium or creatinine or in cases of drug intolerance.

At baseline, each patient underwent assessment of BCVA on standard ETDRS charts, converted into logarithm of the minimal angle of resolution (logMAR) for statistical analysis, along with comprehensive ophthalmological examination and multimodal imaging by fundus autofluorescence, FA, ICGA and spectral domain-OCT (SD-OCT; Spectralis HRA+OCT [Heidelberg Retina Angiograph + OCT];
Heidelberg Engineering, Heidelberg, Germany) and swept-source OCT-A (PLEX Elite 9000; Carl Zeiss Meditec Inc., Dublin, CA, USA). Patients included in the study were followed up at 1 and 3 months with BCVA, IOP, biomicroscopy, SDOCT and OCT-A examinations.

SD-OCT evaluation included CMT analysis using macular thickness maps. Manual measurement of subfoveal retinal fluid (SRF) height was performed for each control, measuring the distance between the end of the outer segment and the retinal pigment epithelium at the foveal center. The subfoveal choroidal thickness (CT), defined as the vertical distance between the hyper-reflective line of Bruch's membrane and the chorio-scleral interface, was measured manually using an enhanced-depth imaging (EDI) technique.

The main outcome measures included comparisons of BCVA, CMT, SRF and subfoveal CT between the treatment and control groups and at all time points. Safety analysis and treatment tolerance were recorded at each visit.

\section{Statistical Analysis}

Statistical analyses were performed using SPSS Statistics version 20 software (IBM Corp., Armonk, NY, USA). Results of descriptive analyses were expressed as means \pm standard deviations for quantitative variables, and as counts and percentages for categorical variables. Associations between categorical variables were analyzed using Fisher's exact test. The Gaussian distribution of continuous variables was verified with the Kolmogorov-Smirnov test. Comparisons of age, CSC duration, BCVA, CMT, SRF and subfoveal CT between treatment and control groups were performed using the Student independent samples $t$ test. Comparisons of BCVA, SRF, CMT and subfoveal CT between the three time points were performed using repeated measures analysis of variance (ANOVA) with Bonferroni correction for post hoc analysis. In all analyses, $p$ values $<0.05$ were considered statistically significant.

\section{RESULTS}

A total of 27 eyes of 27 white patients met the inclusion criteria and were included in the 
study. Fifteen eyes of 15 patients ( 2 female, 13 male) were included in the treatment group, while 12 eyes of 12 patients ( 1 female, 11 male $[p=1.000]$ ) were included in the control group. The subjects of the two groups were similar in age, with a mean age of $44 \pm 9$ years (range $30-65$ years) and $47 \pm 11$ years (range 28-66 years, $p=0.493$ ) for the treatment and control groups, respectively. No patients in either group were affected by any condition that might play a role in the development of CSC, including medication use (such as steroids), pregnancy or other systemic diseases (e.g. endogenous hypercortisolism) related to the disease pathogenesis. In particular, no included patients were receiving any type of chronic therapy.

Table 1 Demographics and main clinical features of the study population

\begin{tabular}{|c|c|c|c|}
\hline & $\begin{array}{l}\text { Treatment } \\
\text { group }\end{array}$ & $\begin{array}{l}\text { Control } \\
\text { group }\end{array}$ & $P$ value \\
\hline Eyes (patients), no. & $15(15)$ & $12(12)$ & \\
\hline \multicolumn{4}{|l|}{ Sex, no. } \\
\hline Male & 13 & 11 & \\
\hline Female & 2 & 1 & $1.000^{\mathrm{a}}$ \\
\hline $\begin{array}{l}\text { Age, years } \\
\quad(\text { mean } \pm S D)\end{array}$ & $44 \pm 9$ & $47 \pm 11$ & $0.493^{\mathrm{b}}$ \\
\hline $\begin{array}{l}\text { Duration of } \\
\text { symptoms, weeks } \\
\text { (mean } \pm \text { SD) }\end{array}$ & $6.9 \pm 3.1$ & $6.3 \pm 2.8$ & $0.645^{\mathrm{b}}$ \\
\hline $\begin{array}{c}\text { BCVA, logMAR } \\
(\text { mean } \pm S D)\end{array}$ & $0.15 \pm 0.12$ & $0.12 \pm 0.06$ & $0.337^{\mathrm{b}}$ \\
\hline $\begin{array}{l}\mathrm{CMT}, \mu \mathrm{m} \\
\quad(\text { mean } \pm \mathrm{SD})\end{array}$ & $426 \pm 104$ & $409 \pm 113$ & $0.693^{b}$ \\
\hline $\begin{array}{l}\mathrm{SRF}, \mu \mathrm{m} \\
\quad(\text { mean } \pm \mathrm{SD})\end{array}$ & $188 \pm 107$ & $171 \pm 100$ & $0.679^{\mathrm{b}}$ \\
\hline $\begin{array}{l}\text { Subfoveal CT, } \mu \mathrm{m} \\
\quad(\text { mean } \pm \mathrm{SD})\end{array}$ & $436 \pm 78$ & $378 \pm 76$ & $0.064^{\mathrm{b}}$ \\
\hline
\end{tabular}

$S D$ standard deviation, $B C V A$ best-corrected visual acuity, $C M T$ central macular thickness, $S R F$ subretinal fluid, $C T$ choroidal thickness

${ }^{a}$ Fisher's exact test, ${ }^{\text {b}}$ Student's $t$ test
At baseline, patients had been affected by CSC for a mean of $6.6 \pm 2.9$ weeks (median 6; range 1-11 weeks) and presented a leakage point at ICGA. In accordance with the inclusion criteria, no signs of CNV were revealed on OCTA or dye angiography. On structural SD-OCT, all patients presented SRF (mean SRF, $180 \pm 102 \mu \mathrm{m}$; range, $21-363 \mu \mathrm{m})$, and BCVA was $0.14 \pm 0.10$ logMAR (range 0.0-0.4). As reported in Table 1, no differences in CSC duration, BCVA, CMT, SRF or subfoveal CT were noted at baseline between the two groups.

In the treatment group, BCVA improved significantly during the follow-up, from $0.15 \pm 0.12 \log$ MAR at baseline to $0.10 \pm 0.11$ logMAR after 1 month $(p=0.018)$, and to $0.06 \pm 0.06 \log$ MAR after 3 months $(p=0.011)$. BCVA also improved in the control group, although this was not statistically significant (from $0.12 \pm 0.06 \log$ MAR at baseline to $0.09 \pm 0.05 \log$ MAR after 1 month $[p=0.246]$, and to $0.08 \pm 0.07$ logMAR after 3 months $[p=0.498])$. However, no significant difference was observed between the two groups in BCVA at baseline or at the 1- or 3-month follow-up $(p=0.337, \quad p=0.816$ and $p=0.378$, respectively).

At the first follow-up visit after 1 month, 7 of 15 eyes (47\%) in the treatment group demonstrated complete SRF resolution on structural SD-OCT, while only 1 of 12 eyes $(8 \%)$ in the control group achieved the same result $(p=0.043$; Figs. 1,2$)$. A significant difference was also evident at the second follow-up visit, after 3 months: 12 of 15 eyes (80\%) in the treatment group demonstrated complete SRF resolution (Fig. 1), versus 3 of 12 eyes (25\%) in the control group $(p=0.007)$. In the treatment group, SRF decreased significantly during the follow-up (SRF: $188 \pm 107 \mu \mathrm{m}, \quad 78 \pm 85 \mu \mathrm{m}$ $[p=0.014]$ and $17 \pm 50 \mu \mathrm{m}[p<0.001]$ at baseline and 1- and 3-month follow-up, respectively), and CMT also decreased significantly, from $426 \pm 104 \mu \mathrm{m}$ at baseline to $330 \pm 81 \mu \mathrm{m}$ at 1-month follow-up $(p=0.028)$ and to $274 \pm 60 \mu \mathrm{m}$ at 3-month follow-up $(p<0.001$; Table 2). In the control group, SRF decreased slightly during follow-up, with no statistically significant difference, and CMT did not change significantly (Table 2). No significant difference 

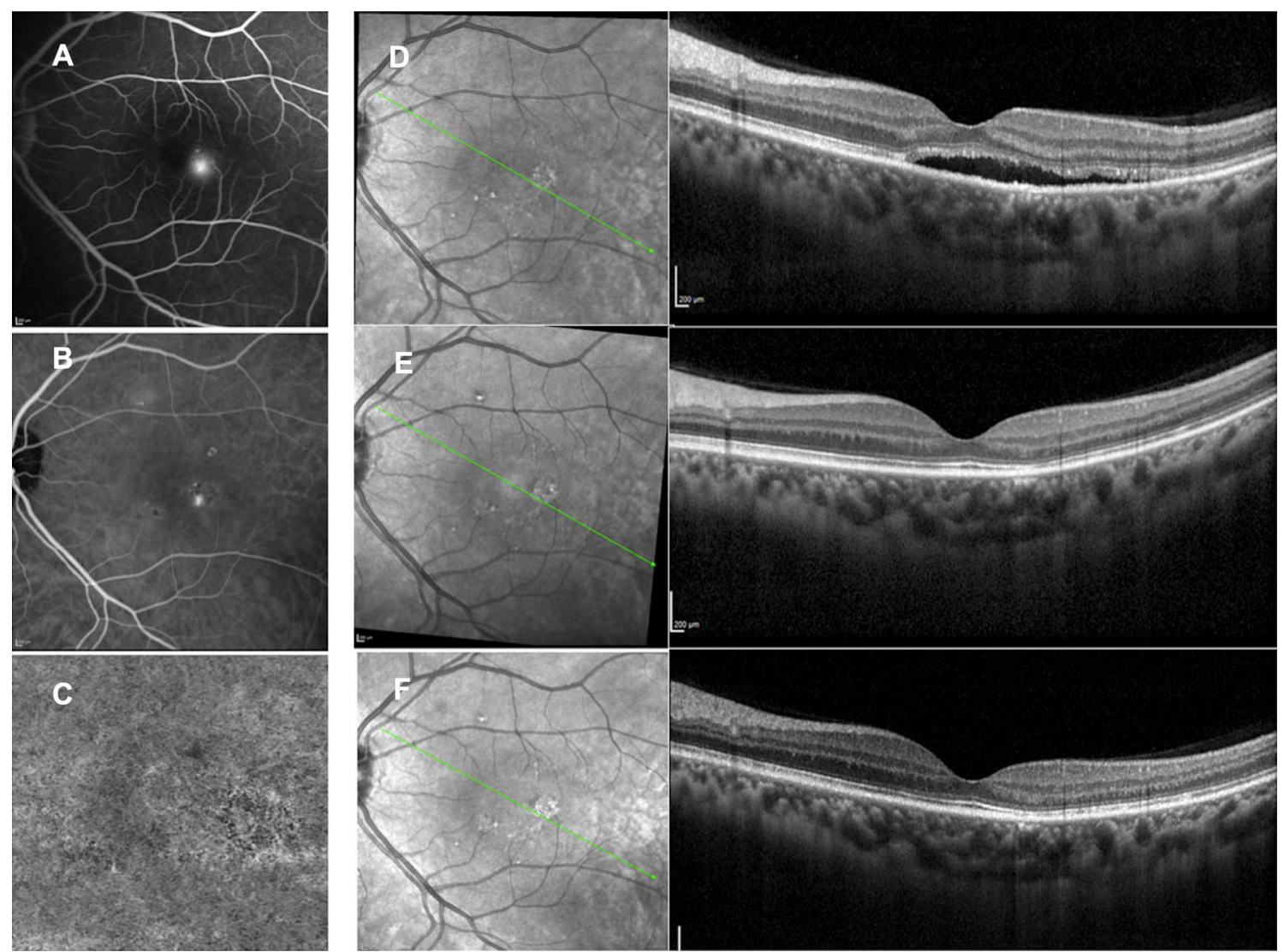

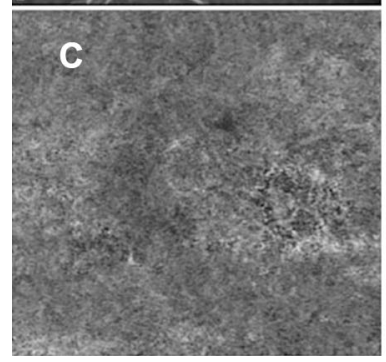

Fig. 1 Multimodal imaging evaluation of a patient affected by acute CSC included in the treatment group. At baseline, fluorescein angiography (a) and indocyanine green angiography $(\mathbf{b})$ revealed a leakage point; $3 \times 3$ en face optical coherence tomography (OCT) angiography of choriocapillaris slab (c) showed no signs of choroidal

was observed between the two groups in SRF or CMT at baseline or after 1 month $(p=0.679$ and $p=0.083$ for SRF at baseline and 1-month follow-up, respectively, and $p=0.693$ and $p=0.079$ for CMT at baseline and 1-month follow-up, respectively). However, a significant difference in SRF and CMT was observed between groups at 3 months $(p=0.002$ and $p<0.001$, respectively).

Subfoveal CT decreased significantly during the follow-up in the treatment group (from $436 \pm 78 \mu \mathrm{m}$ at baseline to $418 \pm 86 \mu \mathrm{m}$ at 3 -month follow-up $[p=0.019]$ ), while no significant decrease was found in the control group ( $p=1.000)$. Furthermore, no differences were found in subfoveal CT between 15 patients who achieved complete resolution of SRF and neovascularization; and structural OCT (d) revealed the presence of subretinal fluid and increased choroidal thickness. At 1 month (e) and 3 months (f) after eplerenone treatment, the complete absence of subretinal fluid was clearly visible on structural OCT

12 patients who did not $(p=0.873, p=0.607$ and $p=0.970$ at baseline and at 1- and 3-month follow-up, respectively).

No serious ocular or systemic side effects related to eplerenone treatment were recorded during the follow-up.

\section{DISCUSSION}

Treatment of CSC remains challenging. Acute CSC is generally a self-limiting disease, with almost $25 \%$ of patients experiencing complete resolution of symptoms in 3 months or less without any treatment [29]. According to the natural benign course of the disease, observation may be considered as a first line of therapy. 

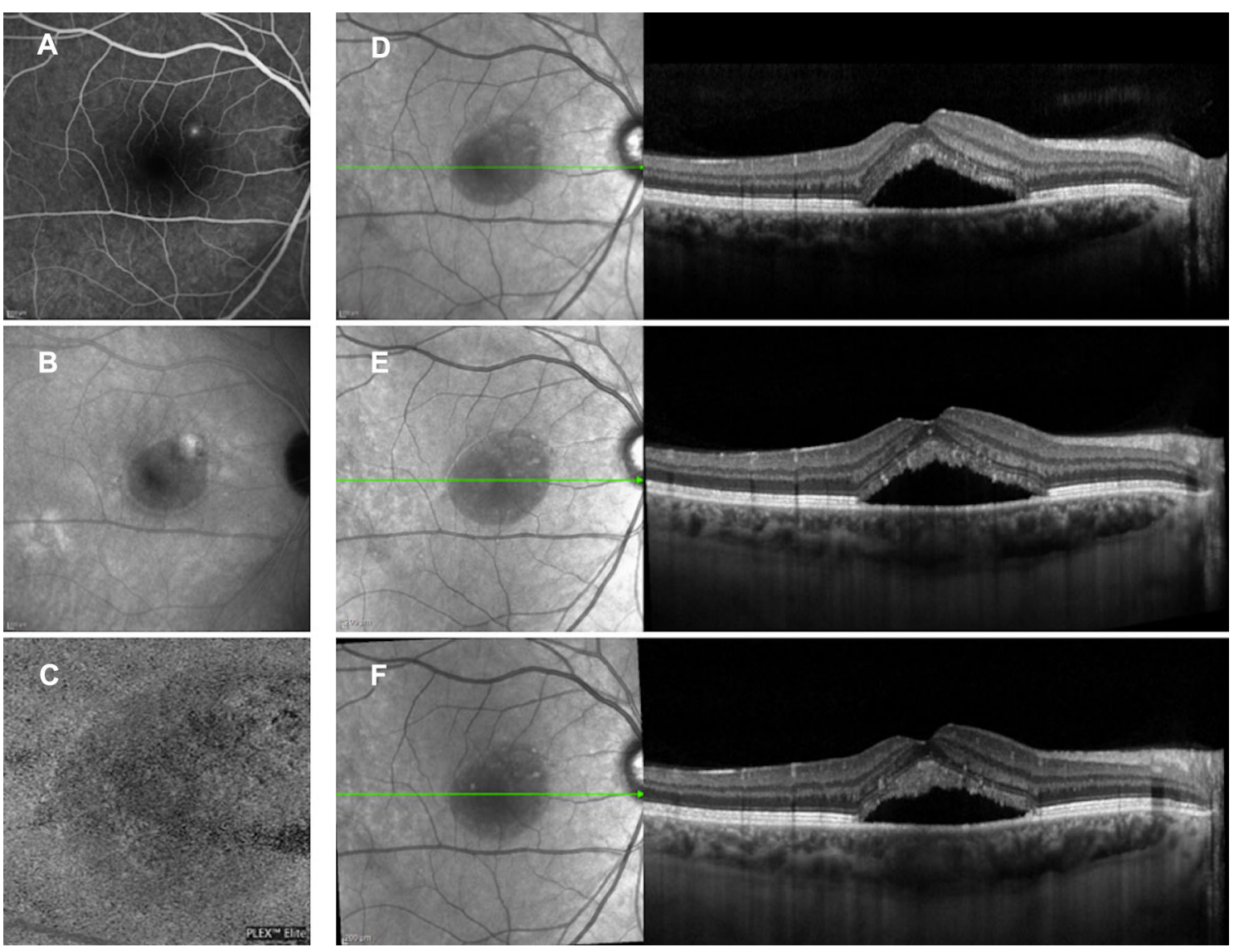

Fig. 2 Baseline multimodal imaging of a patient affected by acute CSC included in the control group. At baseline, fluorescein angiography (a) and indocyanine green angiography (b) revealed a leakage point; $3 \times 3$ en face optical coherence tomography (OCT) angiography of choriocapillaris slab (c) showed no signs of choroidal

Nevertheless, patients who do not achieve spontaneous recovery can progress to chronic disease, with persistent subretinal fluid, progressive RPE damage and irreversible visual deterioration. In addition, since patients generally express a strong desire for faster resolution of symptoms, treatment is also necessary in cases of acute self-limiting disease.

Focal laser photocoagulation targeted to the leakage point has been used in the past to reduce a unique and extrafoveal RPE leak [30]. Full or half-fluence photodynamic therapy (PDT) still plays a key role in the treatment of CSC in order to reduce choroidal hyperpermeability. Several studies have confirmed the efficacy of PDT, showing a reduction in serous neovascularization; and structural OCT (d) revealed the presence of subretinal fluid and increased choroidal thickness. At 1 month (e) and 3 months (f) after observation, no significant differences in subfoveal fluid were observed at structural OCT

retinal detachment and a decrease in choroidal thickness, but the safety effects are still debated over the long term [27-32]. Intravitreal antivascular endothelial growth factor (VEGF) agents have been given to reduce vascular permeability, although the exact role of VEGF in the pathogenesis of CSC is still unclear [33]. Therefore, a specific treatment targeted to the mechanism of choroidal dysfunction is still lacking.

Previous studies have shown that over-activation of the MR pathway is involved in the pathogenesis of CSC, related to an excess of corticosteroids or MR hypersensitivity, based on genetic or environmental predisposition [17]. 
Table 2 Main changes in clinical parameters from baseline at each follow-up in all data

\begin{tabular}{|c|c|c|c|c|c|}
\hline & Baseline & 1-month examination & $p$ value & 3-month examination & $p$ value \\
\hline \multicolumn{6}{|c|}{ BCVA, logMAR (mean \pm SD) } \\
\hline Treatment group & $0.15 \pm 0.12$ & $0.10 \pm 0.11$ & $0.018^{\mathrm{a}}$ & $0.06 \pm 0.06$ & $0.011^{\mathrm{a}}$ \\
\hline Control group & $0.12 \pm 0.06$ & $0.09 \pm 0.05$ & $0.246^{\mathrm{a}}$ & $0.08 \pm 0.07$ & $0.498^{\mathrm{a}}$ \\
\hline \multicolumn{6}{|c|}{$\mathrm{SRF}, \mu \mathrm{m}($ mean $\pm \mathrm{SD})$} \\
\hline Treatment group & $188 \pm 107$ & $78 \pm 85$ & $0.014^{\mathrm{a}}$ & $17 \pm 50$ & $<0.001^{\mathrm{a}}$ \\
\hline Control group & $171 \pm 101$ & $147 \pm 115$ & $0.431^{\mathrm{a}}$ & $134 \pm 118$ & $0.453^{\mathrm{a}}$ \\
\hline \multicolumn{6}{|c|}{$\mathrm{CMT}, \mu \mathrm{m}($ mean $\pm \mathrm{SD})$} \\
\hline Treatment group & $426 \pm 104$ & $330 \pm 81$ & $0.028^{\mathrm{a}}$ & $274 \pm 60$ & $p<0.001^{a}$ \\
\hline Control group & $409 \pm 113$ & $396 \pm 107$ & $1.000^{\mathrm{a}}$ & $409 \pm 103$ & $1.000^{\mathrm{a}}$ \\
\hline \multicolumn{6}{|c|}{ Subfoveal CT, $\mu \mathrm{m}($ mean \pm SD $)$} \\
\hline Treatment group & $436 \pm 78$ & $429 \pm 88$ & $1.000^{\mathrm{a}}$ & $418 \pm 86$ & $0.019^{\mathrm{a}}$ \\
\hline Control group & $378 \pm 76$ & $381 \pm 63$ & $1.000^{\mathrm{a}}$ & $384 \pm 83$ & $1.000^{\mathrm{a}}$ \\
\hline
\end{tabular}

$B C V A$ best-corrected visual acuity, $S D$ standard deviation, $S R F$ subretinal fluid, $C M T$ central macular thickness, $C T$ choroidal thickness

${ }^{a}$ Analysis of variance (ANOVA) with Bonferroni correction for post hoc analysis

The results of this study revealed that oral eplerenone, acting on the MR pathway, was more effective than observation in the treatment of acute CSC. In fact, in the treatment group, BCVA improved significantly at all time points, while visual improvement was not statistically significant in the control group. The results concerning changes in SRF and CMT followed the same trend, with a significant decrease at all follow-up visits in the treatment group, while no statistically significant changes were shown in the control group. With regard to SRF resolution, at 1 month, $47 \%$ of the patients in the treatment group demonstrated complete reabsorption, compared to only $8 \%$ of those in the control group. A significant difference was also evident at 3 months: $80 \%$ of subjects in the treatment group demonstrated complete SRF resolution, versus only $25 \%$ in the control group.

In addition, subfoveal CT decreased significantly during follow-up in the treatment group, while no significant changes were found in the control group. These results strongly support the theory that eplerenone has a meaningful effect against the inappropriate activation of the mineralocorticoid receptors found in the choroidal vasculature of patients with CSC.

The percentage of patients achieving resolution of the disease varies in the literature, according to the main outcome measures considered and the different populations $[18,21,23,24,28]$. In the study by Ghadiali et al. [19], the efficacy of both eplerenone and spironolactone was assessed in eyes with predominantly chronic disease. Even though a significant visual improvement was achieved in all patients, CMT and subfoveal CT showed no significant change. More than $90 \%$ of eyes exhibited chronic CSC, and about half of the patients enrolled had exudative CSC without subretinal fluid, suggesting that both MR antagonists might be less effective in the chronic form of the disease.

However, to the best of our knowledge, no study has analyzed the effects of eplerenone in acute CSC. Sun et al. [29] investigated the effects of spironolactone versus observation in patients with acute CSC. Similar to our study, they found that complete resolution of SRF was 
achieved at 2 months in $56 \%$ of treated patients, compared to $8 \%$ of patients in the control group. The mean CMT and SRF decreased significantly at each visit in both groups, and BCVA improved in both groups at 2 months. In accord with our results, they observed that the subfoveal CT decreased significantly in the treatment group at 2 months, while the change from baseline was not significant in the control group. In addition, the authors reported that none of the patients in the treatment group developed any side effects related to the use of spironolactone, likely because of the short period of treatment. Although eplerenone has a lower affinity for the MR and a theoretically less effective action than spironolactone, our results are in line with those of Sun et al.

We acknowledge that our study has several limitations, primarily related to the small sample and short follow-up duration. Further randomized placebo-controlled clinical studies are needed to increase our knowledge in the treatment of such complex disease.

\section{CONCLUSIONS}

This study showed that patients affected by acute CSC treated with eplerenone achieved higher and faster resolution of the disease than those with observation. Although this study has some limitations, our results show that eplerenone is an effective non-invasive treatment option for acute CSC, with a good safety profile, confirmed by regular laboratory checks. In conclusion, eplerenone may represent an attractive new first-line treatment option for acute CSC.

\section{ACKNOWLEDGEMENTS}

Funding. No funding or sponsorship was received for this study or publication of this article.

Authorship. All named authors meet the International Committee of Medical Journal
Editors (ICMJE) criteria for authorship for this manuscript, take responsibility for the integrity of the work as a whole, and have given final approval for the version to be published.

Disclosures. Ilaria Zucchiatti, Riccardo Sacconi, Eliana Costanzo, Lea Querques and Daniela Montorio have nothing to disclose. Maria Cristina Parravano is a consultant for Allergan, Inc. (Irvine, CA, USA), Bayer Schering Pharma (Berlin, Germany) and Novartis (Basel, Switzerland). Francesco Bandello is a consultant for Alcon (Fort Worth, TX, USA), Alimera Sciences (Alpharetta, GA, USA), Allergan, Inc. (Irvine, CA, USA), Farmila-Thea (Clermont-Ferrand, France), Bayer Schering Pharma (Berlin, Germany), Bausch + Lomb (Rochester, NY, USA), Genentech (San Francisco, CA, USA), Hoffmann-La Roche (Basel, Switzerland), Novagali Pharma (Évry, France), Novartis (Basel, Switzerland), Sanofi-Aventis (Paris, France), ThromboGenics (Leuven, Belgium) and Zeiss (Dublin, CA, USA). Giuseppe Querques is a consultant for Alimera Sciences (Alpharetta, GA USA), Allergan, Inc. (Irvine, CA, USA), Bayer Schering Pharma (Berlin, Germany), Heidelberg (Germany), Novartis (Basel, Switzerland), Sandoz (Berlin, Germany) and Zeiss (Dublin, CA, USA).

Compliance with Ethics Guidelines. All procedures performed in studies involving human participants were in accordance with the ethical standards of the institutional research committee and with the 1964 Helsinki declaration and its later amendments or comparable ethical standards. Informed consent was obtained from all individual participants included in the study.

Data Availability. The datasets generated during and/or analyzed during the current study are available from the corresponding author on reasonable request.

Open Access. This article is distributed under the terms of the Creative Commons Attribution-NonCommercial 4.0 International License (http://creativecommons.org/licenses/ by-nc/4.0/), which permits any noncommercial use, distribution, and reproduction 
in any medium, provided you give appropriate credit to the original author(s) and the source, provide a link to the Creative Commons license, and indicate if changes were made.

\section{REFERENCES}

1. Kitzmann AS, Pulido JS, Diehl NN, et al. The incidence of central serous chorioretinopathy in Olmsted County, Minnesota, 1980-2002. Ophthalmology. 2008;115:169-73.

2. Liew G, Quin G, Gillies M, et al. Central serous chorioretinopathy: a review of epidemiology and pathophysiology. Clin Exp Ophthalmol. 2013;41:201-14.

3. Wang M, Munch IC, Hasler PW, Prunte C, Larsen M. Central serous chorioretinopathy. Acta Ophthalmol. 2008;86:126-45.

4. Loo RH, Scott IU, Flynn HW, et al. Factors associated with reduced visual acuity during long-term follow-up of patients with idiopathic central serous chorioretinopathy. Retina. 2002;22:19-24.

5. Wang MS, Sander B, Larsen M. Retinal atrophy in idiopathic central serous chorioretinopathy. Am J Ophthalmol. 2002;133:787-93.

6. Yannuzzi LA. Type-A behavior and central serous chorioretinopathy. Retina. 1987;7:111-31.

7. Tewari HK, Gadia R, Kumar D, Venkatesh P, Garg SP. Sympathetic-parasympathetic activity and reactivity in central serous chorioretinopathy: a case-control study. Invest Ophthalmol Vis Sci. 2006;47:3474-8.

8. Tittl MK, Spaide RF, Wong D, et al. Systemic findings associated with central serous chorioretinopathy. Am J Ophthalmol. 1999;128:63-8.

9. Spaide RF, Hall L, Haas A, et al. Indocyanine green videoangiography of older patients with central serous chorioretinopathy. Retina. 1996;16:203-13.

10. Iida T, Kishi S, Hagimura N, Shimizu K. Persistent and bilateral choroidal vascular abnormalities in central serous chorioretinopathy. Retina. 1999;19:508-12.

11. Imamura $Y$, Fujiwara $T$, Margolis R, Spaide RF. Enhanced depth imaging optical coherence tomography of the choroid in central serous chorioretinopathy. Retina. 2009;29:1469-73.
12. Jirarattanasopa $\mathrm{P}$, Ooto $\mathrm{S}$, Tsujikawa $\mathrm{A}$, et al. Assessment of macular choroidal thickness by optical coherence tomography and angiographic changes in central serous chorioretinopathy. Ophthalmology. 2012;119:1666-78.

13. Maruko I, Iida T, Sugano Y, et al. Subfoveal choroidal thickness in fellow eyes of patients with central serous chorioretinopathy. Retina. 2011;31:1603-8.

14. Carvalho-Recchia CA, Yannuzzi LA, Negrao S, et al. Corticosteroids and central serous chorioretinopathy. Ophthalmology. 2002;109:1834-7.

15. Haimovici R, Rumelt S, Melby J. Endocrine abnormalities in patients with central serous chorioretinopathy. Ophthalmology. 2003;110:698-703.

16. Haimovici R, Koh S, Gagnon DR, et al. Risk factors for central serous chorioretinopathy: a case-control study. Ophthalmology. 2004;111:244-9.

17. Zhao M, Célérier I, Bousquet E, et al. Mineralocorticoid receptor is involved in rat and human ocular chorioretinopathy. J Clin Invest. 2012;122:2672-9.

18. Bousquet E, Beydoun T, Zhao M, Hassan L, Offret O, Behar-Cohen F. Mineralocorticoid receptor antagonism in the treatment of chronic central serous chorioretinopathy: a pilot study. Retina. 2013;33:2096-102.

19. Ghadiali Q, Jung JJ, Yu S, Patel SN, Yannuzzi LA. Central serous chorioretinopathy treated with mineralocorticoid antagonists: a one-year pilot study. Retina. 2016;36:611-8.

20. Breukink MB, den Hollander AI, Keunen JE, Boon CJ, Hoyng CB. The use of eplerenone in therapyresistant chronic central serous chorioretinopathy. Acta Ophthalmol. 2014;92:e488-90.

21. Leisser C, Hirnschall N, Hackl C, Findl O. Eplerenone in patients with chronic recurring central serous chorioretinopathy. Eur J Ophthalmol. 2015. https://doi.org/10.5301/ejo.5000727.

22. Daruich A, Matet A, Dirani A, et al. Oral mineralocorticoid-receptor antagonists: real-life experience in clinical subtypes of nonresolving central serous chorioretinopathy with chronic epitheliopathy. Transl Vis Sci Technol. 2016;5:2.

23. Singh RP, Sears JE, Bedi R, Schachat AP, Ehlers JP, Kaiser PK. Oral eplerenone for the management of chronic central serous chorioretinopathy. Int J Ophthalmol. 2015;8:310-4.

24. Chin EK, Almeida DR, Roybal CN, et al. Oral mineralocorticoid antagonists for recalcitrant central 
serous chorioretinopathy. Clin Ophthalmol. 2015;9:1449-56.

25. Salz DA, Pitcher JD 3rd, Hsu J, et al. Oral eplerenone for treatment of chronic central serous chorioretinopathy: a case series. Ophthalmic Surg Lasers Imaging Retina. 2015;46:439-44.

26. Kapoor KG, Wagner AL. Mineralocorticoid antagonists in the treatment of central serous chorioretinopathy: a comparative analysis. Ophthalmic Res. 2016;56:17-22.

27. Rabiolo A, Zucchiatti I, Marchese A, et al. Multimodal retinal imaging in central serous chorioretinopathy treated with oral eplerenone or photodynamic therapy. Eye. 2017. https://doi.org/ 10.1038/eye.2017.290.

28. Sun X, Shuai Y, Fang W, et al. Spironolactone versus observation in the treatment of acute central serous chorioretinopathy. Br J Ophthalmol. 2017. https://doi.org/10.1136/bjophthalmol-2017311096.
29. Daruich A, Matet A, Marchionno L, et al. Acute central serous chorioretinopathy: factors influencing episode duration. Retina. 2017;37:1905-15.

30. Robertson DM, Ilstrup D. Direct, indirect, and sham laser photocoagulation in the management of central serous chorioretinopathy. Am J Ophthalmol. 1983;95:457-66.

31. Maruko I, Iida T, Sugano Y, et al. Subfoveal choroidal thickness after treatment of central serous chorioretinopathy. Ophthalmology. 2010;117:1792-9.

32. Chan W-M, Lai TYY, Lai RYK, et al. Half-dose verteporfin photodynamic therapy for acute central serous chorioretinopathy: one-year results of a randomized controlled trial. Ophthalmology. 2008;115:1756-65.

33. Lu HQ, Wang EQ, Zhang T, et al. Photodynamic therapy and anti-vascular endothelial growth factor for acute central serous chorioretinopathy: a systematic review and meta-analysis. Eye. 2016;30:15-22. 